\title{
Research Progress in Anion Functional Fiber and Textile
}

\author{
Zhang Kaijun ${ }^{1}$, Li Qingshan ${ }^{1, *}$, Hong Wei ${ }^{2}$, Wang Yu ${ }^{1}$ \\ ${ }^{1}$ State Key Laboratory of Metastable Materials Science and Technology, Yanshang University, Qinhuangdao, China \\ ${ }^{2}$ People's Republic of China Qinhuangdao Entry-Exit Inspection and Quarantine Bureau Coal Inspection Technique Center, Qinhuangdao, \\ China
}

Email address:

1690143999@qq.com (Zhang Kaijun), liqs126@126.com (Li Qingshan)

${ }^{*}$ Corresponding author

\section{To cite this article:}

Zhang Kaijun, Li Qingshan, Hong Wei, Wang Yu. Research Progress in Anion Functional Fiber and Textile. Science Journal of Public Health. Vol. 4, No. 6, 2016, pp. 494-499. doi: 10.11648/j.sjph.20160406.23

Received: November 4, 2016; Accepted: November 22, 2016; Published: November 24, 2016

\begin{abstract}
Nowadays, anion fibers have been developed as a new kind of functional fiber. Because of the benefits from anions, anion textiles have attracted more and more attention recently. The relationship between the anion and human health was analyzed in the article, and the application and research of anion functional fiber and textile are analyzed. Finally the unresolved problems and possible development trends of testing technology of anion functional fiber and textile were proposed.
\end{abstract}

Keywords: Anion, Functional Fiber, Anion Functional Textile, Human Healthy

\section{Introduction}

Nowadays, with the development of people's health and environmental awareness, the air anion has been used as an important standard for air quality and environment evaluation People will feel relaxed and happy when they are in the environment of waterfalls, sea or forest. There is a very important reason is that the air is rich in anions.

Anion fiber is a functional fiber which can release negative ions, and it has important applications in the field of environmental protection and health care. In the 21 st century, the anion textile has been arouse more attention, and its market develop prospects are very broad [1].

\section{The Generating Mechanism of Anion}

In the natural state, each molecule was tested neutral overall. But under the sufficient energy radiation effect of ultraviolet light, cosmic rays, lightning, and other trace elements, the air molecules will lose part of the electrons rotating around the nucleus. The escaped electrons were called free electron with a negative charge. After the atomic losing or gaining electrons, it will form charged particles which were called ions. It will form air negative ions after the escaping free electrons combine with other neutral gas molecules. These molecules which were missed an electron with a positive charge, and we call it a positive ion or cation. As the ions have short life in the air, there have been ions were balanced out, and continue to have new ions. Therefore, the concentration of positive and negative ions in the air constantly changing, maintaining a dynamic balance. As shown in Figure 1 is the structure of water anion $\left[\mathrm{H}_{3} \mathrm{O}^{+}\left(\mathrm{H}_{2} 0\right)_{7}^{-]}\right.$ in the air.

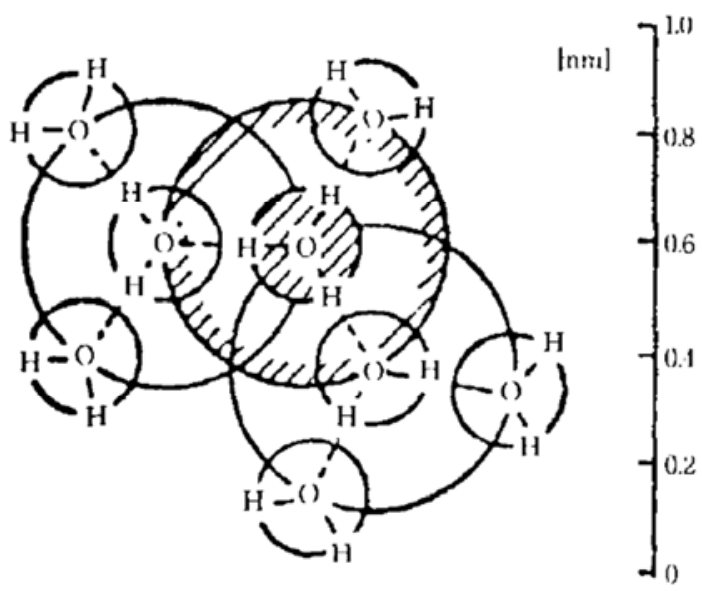

Figure 1. The Structure of Water Anion $\left[\mathrm{H}_{3} \mathrm{O}^{+}\left(\mathrm{H}_{2} \mathrm{O}\right)_{7}{ }_{7}^{-}\right]$in the Air.

Positive and negative ions in the air were divided into large, medium and small ions in accordance with the size of the mobility. The small particle of the negative oxygen ions 
have good biological activity, and they can get through the blood brain barrier into the body exerting their biological effects.[2] According to the US EPA measurement laboratory, the smaller the particle size, the higher the activity, and the more natural diffusion distance away.

\section{The Relationship Between Anion and Human Health}

In 1902, Aschkinass and caspan affirmed the biological significance of air anion. Dr. Philip Lionad, the German physicist, proposed that onions exist in the natural environment are beneficial to human health, and noted that most air anions content in the place of waterfall or valley [3]. There is a certain inactivation effect of anions for microbes, bacteria, viruses, et al [4].

According to the "International Medicine" magazine, the relationship between the contain of onions and human health shows in Table 1:

Table 1. The relation between the contain of the onion and human health.

\begin{tabular}{lll}
\hline Environment & The contain of the onion $\left(\mathbf{c m}^{\mathbf{3}}\right)$ & Relationship \\
\hline Forest and falls & $100000 \sim 500000$ & The body has a natural ability to heal \\
Mountains and seaside & $50000 \sim 100000$ & Bactericidal and inhibiting the spread of disease \\
Countryside and field & $5000 \sim 50000$ & Enhance immunity \\
$\begin{array}{l}\text { City park } \\
\text { Green Street area }\end{array}$ & $1000 \sim 2000$ & Achieve the maintenance of basic health needs \\
Urban residential and closed areas & $100 \sim 200$ & May induce physiological disorders \\
Air conditioning room & $40 \sim 50$ & Induce physiological disorders such as headaches, insomnia neurasthenia, \\
fatigue, etc. \\
\hline
\end{tabular}

Due to the deterioration of the ecological environment and serious pollution, people living in a poor air quality and air composition imbalance in the state, thus affecting people's health. Studies have shown that air pollution caused decline of negative ion concentration in the air. Air negative ions can adsorb, gather and sediment pollutants in the air and refine the air. Anions can destroy cell membrane of bacterial or activity of cell protoplasm and activity of the enzyme, reaching the effect of antimicrobial and bactericidal.

Ions in the air include positive ions and negative ions, modern medical studies have shown that the positive ions are mostly positively charged mineral ions and atmospheric nitrogen, oxygen and other positive ions and organic and water complex positive ions. The places where are more positive ions and where higher prevalence rate of people and more traffic accidents occur. The negative ions are mostly oxygen and hydrated hydroxyl ions, negative ions are beneficial to people, where there are more negative ions, people there are more healthy and longevity. With the research and development of negative ions and health, negative ions were known as "the air of vitamin". Therefore, the amount of negative ions in the air has a direct effect on human health. According to research, negative ions have effect on the human body's 7 system and nearly 30 kinds of diseases with inhibition, mitigation and adjuvant therapy, especially for human health care more apparent.

\subsection{The Effect of Anions on Human Nervous System}

Anion can make the cerebral cortex function and mental activity to strengthen, spirits, work efficiencily, and improve sleep quality. Meanwhile, anions can also strengthen the brain tissue oxidation process, and thus the brain tissue could get more oxygen.

\subsection{The Effect of Anion on Human Cardiovascular System}

According to the observation of scholars, negative ions have significant role in the expansion of blood vessels, and arterial spasm can be lifted to achieve the purpose of lowering blood pressure. Anion is also good in improving cardiac function and improve myocardial nutrition, and it is conducive to hypertension and cardiovascular disease patients restore.

\subsection{The Effect of Anions on Human Blood System}

Studies have confirmed that negative ions could slow down the blood coagulation flow rate, prolong the role of clotting time, and increase the oxygen content in the blood, so it is conducive to oxygen delivery, absorption and utilization. Negative oxygen ions can enter into the blood and directly affect the blood cells of charged particles in the composition and distribution, thus promoting red blood cells, reticulocytes, hemoglobin, calcium increases, and lowering blood sugar, blood lipids and blood viscosity.

\subsection{The Effect of Anions on Human Respiratory System}

Negative ions on the respiratory system the most obvious. This is because the negative ions go into the body are through the respiratory tract, it can improve people's lung capacity. It has been tested that in a glass mask inhalation of negative ions for 30 minutes, the lungs can absorb oxygen increased by $20 \%$, while the amount of carbon dioxide can be increased by $14.5 \%$, so the anion has improved and increased lung function.

\subsection{The Effect of Anions on Human Metabolic System}

Anion could promote the body's redox process, and activate the enzyme system in vivo to promote synthesis and storage of vitamins, promote body metabolism, reduce blood 
lactate content, eliminate fatigue, thus improving work efficiency. Through the reflex and humoral effects, negative oxygen ions could expand the coronary artery to increase coronary blood flow, improve myocardial function, adjust the heart rate, relieve angina and return to normal blood pressure.

\subsection{The Effect of Anions on Human Immune System}

Negative ions can enhance the function of mononuclear phagocytes, increase blood antibody levels, improve the body's non-specific immune function and desensitization. The experts found that inhalation of anion for anaphylactic shock animal can reduce the disease shortened course compared with the control group.

In addition, air anions have sedative and hypnotic effect. If we inhaled the right amount of negative ions every day, there will be great health benefits: energetic, eliminate fatigue and burnout, thus improving work efficiency.

\section{The Applications of Anion Fibers and Textiles}

Anion fiber can release negative ions and effectively improve air quality, and it has been accepted by more and more people. Anion fiber with a certain amount of negative ions additives can continuously generate negative ions through the contact with air and moisture [5]. Such additives also has excellent far-infrared emission function and antibacterial features that make the applications of anion fiber and textile more widely [6-7].

\subsection{Filtration Anion Textiles}

Anion textile has important applications in the filter materials, such as air-conditioning filters, water treatment materials and so on. Anion almost disappeared after a series of air-conditioning air purification treatment, in which long-stay will make people feel chest tightness, fatigue and dizziness. Anion fibers as air-conditioning filters can efficiently clean indoor air and solve the problem above. Filter material with anion fibers for water dispenser filter can kill bacteria in the water, and increase dissolved oxygen in the water. Water treated with anion for growing green plants can improve the survival rate of plants, and shorten the maturity [8].

\subsection{Decoration Anion Textiles}

Anion textiles can be used as indoor upholstery, carpets and curtains, et al. Anion textiles can produce negative ions to eliminate indoor air. Particularly, in the newly renovated houses. There are formaldehyde, benzene, ammonia and other harmful gases left behind after decorating, which reduce harm to human body. Furthermore, cars using anion fiber can remove smell inside the car and purify air inside the vehicle.

\subsection{Healthcare and Medicine Anion Textiles}

With the improvement of people's health requirements of textiles, anion textile has made considerable progress in the health care field. Such textiles can be used as bedding, such as bed sheets, quilt, pillowcases, et al; and anion textile also can be used as gloves, socks and other healthcare fabric. Anion textiles also have huge potential applications in medical supplies, such as used as nursing care, surgical clothing, hygiene products, medical supplies, hospital beds and other supplies. Anion textiles can effectively prevent iatrogenic cross-infection and bacterial infections, and purify the air at the same time.

\section{The Research of Anion Fiber and Textiles}

\subsection{The Processing Method of Anion Fiber and Textiles}

In general, anion textile processing methods can be divided into two categories: one is using anion fibers to produce anion textiles, the other is using solution which containing anion additives after finishing of fabric. After finishing is the use of padding method, coating method or dipping method to have inorganic anion release microparticles fixed on the surface of the fabric, making the fabric has a performance of release anions [9-10]. Liu Yaguang [11] using nano-materials mixed with an special anionic polymer resin, and it was used for oak Simian, and after finishing its far-infrared emission rate is $88 \%$. According to survey, far-infrared emission rate of $80 \%$ or more can play an effective role in health care generally. This method can be applied to a variety of textile products, but there are poor washability, bad feeling and other shortcomings. Different processing methods can affect the release of anion of the anion fiber and textile. In addition, the fibrous fineness, temperature and humidity of the surrounding environment can also influence the release of anion of the fiber. [12]

\subsection{The Research Progress in Anion Generating Materials}

Anion textiles have the ability to release negative ions, because of the addition of anion additives. With the people's attention to anion products, the research of anion additives is also more and more popular with researchers. On the one hand, additives, which have a high-efficiency anion-releasing function, can be obtained by compounding the composite powder. For example, the rare earth compound salt or oxide for the tourmaline dispersion medium can effectively improve the capacity of tourmaline to produce negative air ions. On the other hand, the research on ultrafine processing of negative ion additives is studied. The size of anion additives in the production of fiber have some certain requirements, especially in the production of fine denier fiber process, otherwise, there will be nozzle hole blockage, breakage, floating wire and so on in the silk process. At the same time, anion generation capacity will be improved with the negative ion material particle size refinement. Some of the commonly used negative ion generating materials are described below. 


\subsubsection{Natural ore Which Containing a Trace of Radioactive}

In 1982, Professor Thomas•Mubarak, University of Missouri, studied obtained that these ores contain trace amounts of natural thorium, uranium and other radioactive substances which can release faint radiation, and particles in the air, and in this long-term impact it will be weak ionizing radiation to produce negative ions [13].

\subsubsection{Tourmaline, Wizards Stone, Opal and Other Crystal Materials}

Tourmaline is characterized with boron silicate materials. Wizards stone is an inorganic porous material, and mainly containing silicates, iron, and aluminum oxide. Opal is amorphous or other organic silicate material. Because of this type of ore's thermoelectric and piezoelectric, it can cause potential different change between the mineral crystals when the temperature and pressure of environments have slight variations. The formation of electrostatic voltage can promote the ionization of the surrounding air, and the ionization of electrons combined with adjacent water and oxygen molecules, converted into air anion finally.

\subsubsection{Seabed Sediments, Coral Fossils, Seaweed Charcoal, et al}

Submarine sediments, coral fossils, seaweed charcoal and other substances are the inorganic porous material, with a permanent spontaneous electrode. When subject to external temperature and pressure of small changes, it can make its surrounding air ionization.

\subsubsection{Photocatalyst Material}

Photocatalyst material is photocatalyst. $\mathrm{TiO}_{2}$ is the main component of photocatalyst material, which is a photosensitive semiconductor material. Absorbing ultraviolet light, the energy in the ultraviolet excitation can produce positively charged holes and negatively charged electrons. Positively charged holes and water, negatively charged electrons and oxygen react, respectively. They will produce strong oxidation of hydroxyl radicals which have a bactericidal effect of negative oxygen ions, and at the same time, the free harmful substances in the air and microbial will be decomposed into carbon dioxide and water, thereby purifying the air, sterilization and disinfection.

\subsubsection{Composite Anion Generating Material}

The composite anion generating material is prepared by mixing two or more natural minerals into a material which has the function of releasing anion and far-infrared. Jin Zongzhe [14] had $\mathrm{ZnO}, \mathrm{CaCO}_{3}$ and $\mathrm{Al}(\mathrm{OH})_{3}$ mixed, the anion powder mixture can release of about $1000 / \mathrm{cm}^{3}$ of the negative ions.

\subsection{The Research Status of Anion Fiber and Textiles}

Anion generating materials attracted people's attention gradually from the 90 s last century. In the international market, the anion functional textiles show many characters, such as excellent quality, good function, various types, specifications and more patterns and so on. Japan, which is the earlier starting country in the world, has done a lot of research and develop work in this area. In the Japanese market, anion textiles have had a certain scale, showing a good momentum of development.

In the anion processing materials, the Japanese company KomatsuSeiren has been successfully developed natural mineral fixed technology in the fabrics using finishing technology, and these minerals can produce negative ions, which make people feel good and refreshing. Toray Industries of Japan has studied a new finishing technology "Aquaheal". The finishing technology uses the deep seabed raw materials as an anion-generating material. After finished with Aquaheal, the fabrics can be widely used in work clothes, underwear, sportswear, bedding and home decorations. Japan's bell spinning synthetic fiber company developed Ionsafe acrylic fiber, and the fiber incorporates minerals which can produce negative ions. This mineral is a special ceramic powder, which can continuously generate negative ions when it is kneaded and uniformly dispersed in the fiber.

In the mid-1990s, China has began to develop anion textiles, and coastal areas as the center, and take the product into the interior, as well as the world gradually. However, the development and application of anion textiles in China mainly stay in the stage of chemical fiber, usually using ceramic powder or tourmaline powder and other anion additives to make negative ion functional masterbatch, and then use blending or copolymerization method to modify the fiber. Some companies in China has successfully developed many anion fiber and its textile products, such as the Shanghai (China) Chemical Fiber Co., Ltd.'s anion far-infrared viscose fiber, Chengdu (China) Fuxing health care textile company developed series of anion far-infrared health care textiles and Shanghai Petrochemical Company developed "singular fiber" anion fiber which can be used as a filler of medical textiles, bedding or car seats. [15]

Compared with Japan, whether it is basic research or applied research, China's development and application work of anion textile is still in its infancy. However, China has a unique geographical advantage in Xinjiang (China), Inner Mongolia (China), Guilin (China) and Yunnan (China) and other places, where are rich in mineral resources, and the natural resources to be further development and utilization. With this natural advantage, there will be broad market prospects in in-depth study of anion fibers and develop more functional health anion textiles.

\subsection{The Research Progress of Testing Methods for Anion Functional Textiles}

\subsubsection{The Research Progress of Detection for Textile Anion Concentration}

The anion concentration detection process for anion functional fiber and textile: controlling a certain temperature and humidity conditions first, and then stimulating anion fabric negative ions using generating devices, and last testing the anion concentration using the air anion concentration tester [16]. Chen Yuehua [17] studied the anion concentration 
test method of soybean protein fiber, and they used a similar hand rub friction mechanical transmission system as the negative ion generating device, which can ensure the friction be quantified and stability. Cai Shujun [18] studied the anion function of fabrics, which are fabricated by sol-gel technique. In order to simulate the friction between the textile and the actual wearing process, a flat-type rubbing fastness meter was used as the negative ion exciting device. Through analyzing a large number of test results under the closed and open environment, the test conditions of textile anion release concentration were got. He Xiuling [19] studied the process of friction produced negative ions, the test results show that the occurrence of negative ions will not continue with the unlimited increase physical stimulation of the external role, and its with saturation.

\subsubsection{The Testing Methods for Anion Functional Textiles}

At this stage the domestic textile laboratories use hand rub method and FCL fabric anion test method as textile anion concentration detection methods [20]. Hand rub method is open measurement, the operation is relatively simple, but the error is large; at the same time, the test environment is open, the negative ions will be diluted with the flow of air, and air positive charge and dust and other neutral particles will also neutralize a part of negative ions, leading a certain chance to test results [21]. FCL fabric negative ion test method is a closed measurement, and it is a new method for negative ion textiles production and standard status. The test principle of the method is not fundamentally different from that of the hand rub method, but the test result is more accurate and reproducible. Now the most widely used is the closed test system, which can reduce the error of each measurement to a certain extent.

\subsubsection{The Research Prograss of Testing Standards for Textile Anion Concentration}

At present, there is no standard, complete and unified anion assessment and testing standard in the world. The earliest study of the function of air anions is a Japanese scientist, and the research results are also get the attention of some scientists in other countries quickly. China has done some work in the detection of anion concentration and standard formulation. Although there is no uniform and international recognized standard for assessment of air anions in the world, the development of anion standard work at home and abroad has achieved some results. According to a research survey, it shows that a total of five domestic and international textile anion testing standards, as shown in Table 2:

Table 2. Anion textile testing standards at home and abroad [22].

\begin{tabular}{lll}
\hline Country & Number & Name \\
\hline China & GB/T30128-2013 & Detection and Evaluation of Anions in Textiles \\
China & GB/T18809-2002 & General specification for air ion measuring instrument \\
China & SN/T2558.2-2011 & Testing methods for functional textiles for import and export. Part 2: anion content \\
China & JC/T2040-2010 & Anion functional building interior decoration materials \\
Japan & JIS B 9929-2006 & Measuring method of air anion \\
\hline
\end{tabular}

\section{Conclusion and Outlook}

With the improvement of people's living standards, the requirements of the textile products from the aesthetic enhance to the functional care. The development of anion fiber technology can not only meet people's health and environmental protection needs, but also led development and progress of the textile industry. With the understanding of the mechanism of air anions and the positive effect of air anions on human health, the development of anion textiles is on an upward trend, and its technology development potential is great, but there are also many imperfections and problems to be solved. The next step should focus on two aspects, one is superfine and high efficiency anion powder additive's preparation and the other is the improvement of anion testing system. With people's understanding of the mechanism and the preparation technology of anions, it is believed that anion fiber and its textile products will have a high economic value and broad market prospects.

\section{Acknowledgements}

The authors are grateful for State Key Laboratory of
Metastable Materials Science and Technology of Yanshang University, and the support of People's Republic of China Qinhuangdao Entry-Exit inspection and Quarantine Bureau Coal inspection Technique Center.

\section{References}

[1] Wang Doren. Anion fiber development and application of the progress [J]. Hebei Textile, 2011 (144): 1.

[2] Yun Qiuxia. Discussion on the health mechanism and application of anionic viscose fiber [J]. Synthetic Materials Aging and Application, 2015, 44 (6): 102.

[3] Ma Yunhui. Application of Air Negative Ions [J] Journal of Baoji University of Arts and Sciences, 2010, 30 (1): 42.

[4] Daniell W, Camp J, Horstman S. Trial of a negative ion generator device in remediating problems related to indoor quality $[\mathrm{J}]$. Jourmal Occupational Environmental Medicine, 1991, 33 (6), 681.

[5] Wang Lingling, Li Yabin. Anion functional health fiber and its textile products $[\mathrm{J}]$. Textile of Textile Science and Technology, 2010 (6): 11.

[6] Sun Chao. Anion textiles development and performance research [D]. Shanghai: Donghua University, 2005. 
[7] Duan Julan, Wang Liansheng, Xu Xiaochen. Properties and Application of Anion Fibers [J]. Jinshan Oil Chemical Fiber, 2001 (2): 53.

[8] Qiu Fagui, Li Quanming, Zhang Mei, et al. Research of Anion Fibers and Their Textiles [J]. Hi-Tech Fiber \& Application. 2008, 33 (3): 19.

[9] Ren Cailing, Meng Jiaguang, Wang Jiguo. Test Analysis of Negative Ion Finishing Process of Cotton Fabric [J]. Cotton Textile Technology, 2011, 39 (12): 31.

[10] Wu Jianhua. Far-infrared textile processing technology [J]. Textile Review, 2014 (5): 87.

[11] Liu Yaguang, Yang Muying, Tian Shuo, et al. Study on the processing technology and properties of negative ion far-infrared silk oak silk [J]. Liaoning Silk, 2015 (3): 26.

[12] Wu Ruozi. Development and Application of Anion Home Textiles [J]. Chemical Fiber and Textile Technology, 2011, 40 (1): 41 .

[13] Nissin Spinning Co., Ltd. "Environmental purification, anion, health therapy," the use of radiation-stimulating effect of fiber material [J] Dyeing and finishing, 2005, 4 (247): 42.

[14] Jin Zongzhe, Liang Jinsheng, Wang Jing. Air purification and antimicrobial functional material for rare earth activated negative ion generation and its manufacturing method: CN1242943A [P]. 2000-02-02.
[15] Xiong LiJun, Han ShaoHua, Tang Hao. Research Progress and Influencing Factors of Air Negative Ions [J]. Resources and Environment, 2011, 21 (3): 184-187.

[16] Mo Shiqing, Chen Yanxia, Shi Yidong. Technique and Application of Negative Ions Textiles [J]. Dyeing and finishing, 2010, 32 (5): 42.

[17] Chen Yuehua, Gong Peihu, Yang Shibin. Effects of Negative Ions on Soybean Protein Fibers [J]. Textiles, 2006, 27 (4): 63.

[18] Cai Shujun. Anion textiles testing and sol-gel method for negative ion functional finishing [D]. Shanghai: Donghua University, 2008.

[19] He Xiuling. Study on detection method of textile anion performance $[\mathrm{J}]$. Dyeing and finishing chemicals, 2011, 28 (8): 50 .

[20] Fu Guangwei, Yan Yan, Yang Ping. FCL fabric negative ion test method [J]. Textile Review, 2010 (2): 88.

[21] Xiong Lijun, Han Shaohua, Tang Hao. Research Progress and Influencing Factors of Air Negative Ions [J]. Resources and Environment, 2011, 21 (3): 184.

[22] Zhang Zhuo, Zhou Changzheng, Xie Maozhong. Research progress on the detection of negative ion concentration in functional textiles [J]. Textiles in Textile Science and Technology. 2014 (5): 4. 